\title{
Ultra-short pulse compression using photonic crystal fibre
}

\section{Gail McConnell ${ }^{1}$ and Erling Riis ${ }^{*}$}

1: Centre for Biophotonics, University of Strathclyde, Glasgow, G4 ONR, Scotland

2: Department of Physics, University of Strathclyde, Glasgow, G4 ONG, Scotland

\begin{abstract}
A short section of photonic crystal fibre has been used for ultra-short pulse compression. The unique optical properties of this novel medium in terms of high nonlinearity and relatively small group velocity dispersion are shown to provide an ideal platform for the standard fibre pulse compression technique used directly on the nanoJoule output pulses from a commercial laser system. We report an order of magnitude reduction of the pulse width to 25 fs FWHM but predict a substantially improved performance with a dedicated fibre design. Good agreement is obtained with a simple model for the spectral broadening in the fibre.
\end{abstract}

PACS: 42.65.Re, 42.70.Qs, 42.81.Cn

*FAX: +44 141552 2981, Tel: +44 141548 3490, e-mail: e.riis@strath.ac.uk 


\section{Introduction}

The non-linear optical properties exhibited by optical fibres have been studied widely. Observed effects include four-wave mixing, stimulated Raman and Brillouin scattering, crossphase modulation and self-phase modulation [1]. The latter of these is conceptually the simplest, relying only on the Kerr non-linearity of the medium. Manifestations of this effect include soliton formation [2], pulse compression [3,4] and additive pulse mode-locking [5]. Although the non-linear susceptibility of fused silica is very small, these effects arise from the small mode area and long interaction lengths available with state-of-the-art optical fibres.

Of particular interest for the present work is the development of techniques for optical pulse compression. The key step here is to take advantage of the ability of the wider bandwidth generated by self-phase modulation to support a shorter pulse. A range of implementations have been reported using ordinary step-index fused silica single mode fibre as the non-linear medium. The combination of the self-phase modulation and a positive group velocity dispersion in the fibre results in a spectrally broadened and stretched output pulse with an almost linear frequency chirp across it. By introducing the correct amount of negative dispersion the various frequency components of the pulse can then be made to catch up with each other in time to produce a pulse substantially shorter than the original one. Indeed, compression factors of about two orders of magnitude have been reported [6] as well as output pulses of only a few fs [7]. The required negative dispersion is obtained from either a pair of diffraction gratings, a prism pair, or a combination of the two.

A common feature with the reported implementations of ultra-short pulse compression based on standard silica fibre is the need for pulse energies significantly higher than what is generally obtained directly from a mode-locked laser source. Thus, the record-breaking few fs pulses have been generated from complex low repetition rate amplified or cavity dumped 
systems. A notable exception to this is a recent report [8] on pulse compression of the direct output of a laser source by a factor of $3-6$ using a short section of photonic crystal fibre (PCF). With a mode diameter of only $1.7 \mu \mathrm{m}$ this fibre is highly non-linear.

A similar technique has recently been reported used for compressing the high power (60 W) output of a mode-locked Yb:YAG thin disk laser [9]. In this case a large mode area fibre design (diameter of $16 \mu \mathrm{m}$ ) was chosen to avoid optical damage of the fibre. Although pulse widths as low as $33 \mathrm{fs}$ were observed this choice of fibre has a slight disadvantage of not providing control of the group velocity dispersion.

The pulse lengths reported from a PCF based compressor do not yet compete with those from conventional amplified systems or indeed from highly specialised sub-10 fs laser sources. However, the PCF system has the clear advantage of being a simple and versatile add-on to standard commercial lasers operating in the few hundred fs regime.

In this paper we report on an order of magnitude reduction in the pulse width of the output of a commercial Ti:Sapphire laser using a short section of PCF. A model is developed for the process showing that the unique properties of the fibre in terms of high non-linearity and low dispersion combine to give ideal conditions for ultra-short pulse compression. This result and the described potential for further significant improvements through a dedicated fibre design demonstrate that this technique may provide a simple and effective route to the few femto-second regime where the direct operation of a laser is significantly more challenging.

\section{Photonic crystal fibre}

The development of the photonic crystal fibre [10] has brought renewed interest to the field of non-linear fibre optics. PCFs are micro-structured fibres, where the light is guided by a number 
of periodically arranged air holes extending the length of the fibre. These holes create a photonic bandgap in the transverse dimension resulting, for instance, in fibres that are singlemode throughout the visible range [11]. The superior guiding property also allows for a reduction in the core diameter down to a few microns, leading to a significant increase in the propagating peak intensity. This is of obvious benefit in the study of non-linear effects, but just as significant is the fact that the exact nature of the micro-structure determines the group velocity dispersion (GVD) of the fibre. Typically, the zero-dispersion point, $\lambda_{0}$, in a $1-2 \mu \mathrm{m}$ diameter PCF is shifted from the bulk silica value of around $1270 \mathrm{~nm}$ down to $600-800 \mathrm{~nm}$ $[12,13]$. This means that it is now possible to have a fibre with anomalous dispersion at a convenient input wavelength to accommodate a wider range of commercially available laser sources, such as the Ti:Sapphire laser.

The most spectacular manifestation of this high non-linearity in an anomalously dispersive regime is the generation of a white-light supercontinuum extending well over an optical octave [12]. The detailed mechanisms involved in the supercontinuum generation are complicated [14] but the key is the ability to form solitons at wavelengths above the zero-point for the group velocity dispersion. The associated and well-known effects of soliton selffrequency shift and shedding of energy to shorter wavelengths due to third-order dispersion provide the broadening, while four-wave mixing tends to fill in any remaining gaps [14]. The notable feature of the PCF is that the small mode area brings extreme prominence to these otherwise often subtle effects.

The situation is far simpler for wavelengths below the zero-point for the group velocity dispersion. The normal dispersion prevents soliton formation, leaving self-phase modulation as the main non-linear effect. This is the basis for the PCF pulse compressors reported previously $[8,9]$ and investigated further in this paper. 


\section{Experimental set-up}

A schematic of the experimental set-up is shown in Fig. 1. The commercial Ti:Sapphire laser (Coherent Mira 900-F) emits an $80 \mathrm{MHz}$ train of pulses in the region $720-880 \mathrm{~nm}$. The pulse width was measured to be $\sim 250 \mathrm{fs}$ with an interferometric autocorrelation trace consistent with a sech ${ }^{2}$ pulse shape. This light is focused into a length of PCF. The output is collimated and passed through a dispersion compensating stage consisting of two parallel 600 lines $/ \mathrm{mm}$ gratings separated by $2-12 \mathrm{~mm}$. The first grating is at normal incidence. This grating pair

introduces a negative dispersion in the beam of $0.463\left(\mathrm{ps}^{2} / \mathrm{m}\right) \times b_{0}$, where $b_{0}$ is the separation between the gratings [15]. The light is then sent to an autocorrelator set up as a Michelson interferometer with one arm sweeping $\sim 2 \mathrm{~mm}$ at a rate of $\sim 1 \mathrm{~Hz}$. The combined beam is focused onto an GaAsP photo diode, which has only a two-photon response at wavelengths in the $800 \mathrm{~nm}$ region [16]. Low dispersion mirrors are used throughout the set-up and the beam splitter in the autocorrelator is a $0.5 \mathrm{~mm}$ thick glass plate with a $\sim 50 \%$ reflectivity coating. The autocorrelation signal is sampled for every $\sim 14 \mathrm{~nm}$ change in the path length difference of the two arms.

The photonic crystal fibre is manufactured by Crystal Fibre A/S. It is made of pure silica and has a hexagonal structure of air holes to guide the light in a $2.6 \mu \mathrm{m}$ diameter core. The distance between adjacent air holes is $1.8 \pm 0.2 \mu \mathrm{m}$ and the hole size to separation ratio is 0.35 . This structure gives rise to a high non-linearity and a zero dispersion point around $900 \mathrm{~nm}$. The fibre therefore has a low and positive dispersion throughout the normal Ti:Sapphire operating range. The dispersion is estimated to be $50 \mathrm{ps} / \mathrm{nm} \cdot \mathrm{km}$ at $800 \mathrm{~nm}$. An almost identical fibre has been used to demonstrate supercontinuum generation in the $700-1200 \mathrm{~nm}$ range [17]. 


\subsection{Spectral broadening in PCF}

First we consider the spectral broadening properties of the fibre. Fig. 2 shows an example of the spectrum of the output of the fibre with $188 \mathrm{~mW}$ of light transmitted through $86 \mathrm{~mm}$ of fibre (representing a transmission of $61 \%$ ). This corresponds to a pulse energy of $\sim 2.35 \mathrm{~nJ}$. The laser wavelength is $830 \mathrm{~nm}$. The spectrum shows the multi-peak structure characteristic of selfphase modulation with a broadening to $38 \mathrm{THz}$. The spectral bandwidth is observed to increase linearly with the power transmitted through the fibre as shown in Fig. 3.

In order to gain a better understanding of the spectral broadening process we have modelled the propagation of a pulse through the fibre. Assuming a sech ${ }^{2}$ laser pulse the electric field is given by:

$$
E_{i n}(t)=\exp (-i \omega t) \operatorname{sech}\left(2 \ln (1+\sqrt{2}) t / T_{0}\right)
$$

where $\omega$ is the laser frequency and $T_{0}$ the FWHM of the pulse.

The self-phase modulation introduces an intensity dependent phase shift:

$$
\varphi_{n l}(t)=\varphi_{\max } I(t) / I(0)
$$

where $I(t)$ is the propagating intensity distribution and the maximum phase, $\varphi_{\max }$, is proportional to the length of the fibre and its non-linearity. This rapid variation of the phase across the pulse results in a chirp of the frequency, but no temporal broadening.

In the absence of any dispersion in the fibre the output spectrum is determined simply as the Fourier transform of $E_{\text {in }}(t) \exp \left[i \varphi_{n l}(t)\right]$. This spectrum has a number of maxima which for 
large values of $\varphi_{\max }$ is approximately equal to $\frac{1}{2}+\varphi_{\max } / \pi$, while the overall bandwidth of the output is proportional to $\varphi_{\max } / T_{0}$ [1]. The dotted curve in Fig 2 shows a close match to the experimental data assuming a maximum phase of $8.8 \pi$ and a pulse length of $315 \mathrm{fs}$. This is significantly longer than the measured pulse width. This discrepancy is due to the fact that the experiment operates slightly into the region of normal dispersion. Hence, as the pulse propagates through the fibre and the spectrum broadens, the temporal profile of the pulse also broadens, resulting in an overall decrease in the spectral width. We have modelled this using a split-step Fourier technique [1] assuming a group velocity dispersion of $20 \mathrm{ps} / \mathrm{nm} \cdot \mathrm{km}$ for an 86 $\mathrm{mm}$ long fibre. At this relatively low group velocity dispersion, the qualitative features of a spectrum broadened by self-phase modulation are still dominant. The two main effects on the output spectrum of the introduction of the GVD in the calculation is a slight reduction in the bandwidth of the broadened pulse (by $\sim 17 \%$ in this case) and a flattening of the central part of the spectrum compared with the pure self-phase modulation spectrum. This is in qualitative agreement with the experimental data as presented in Fig. 2, where the simulated output spectrum is shown for a $270 \mathrm{fs}$ pulse propagated through $86 \mathrm{~mm}$ of the $20 \mathrm{ps} / \mathrm{nm} \cdot \mathrm{km}$ fibre, with a maximum phase modulation per unit length $\Delta \varphi_{\max } / \Delta L$ of $1.10 \pi / \mathrm{cm}$.

The dispersive properties for the fibres used in this work are not known accurately. For simplicity and in order to not introduce any more free unknown parameters in the model all the simulations presented in this paper assume a wavelength independent value for the dispersion. While not strictly correct this approximation does reproduce the qualitative features observed in the experiment. The main effect of the introduction of a linearly varying dispersion of a realistic magnitude in the model is that it then predicts the slight asymmetry in the spectrum apparent in Fig. 2. 
The maximum phase can also be determined from first principles. It can be expressed as [1]:

$$
\Delta \varphi_{\max } / \Delta L=\gamma P_{0}
$$

where $P_{0}$ the peak power in the pulse and

$$
\gamma=\frac{2 \pi n_{2}}{\lambda A_{\text {eff }}}
$$

$A_{\text {eff }}$ is the effective mode area in the fibre and $n_{2}$ the non-linear refractive index. Assuming $A_{\text {eff }}$ is the area of a circle with diameter $2.6 \mu \mathrm{m}$ and $n_{2}=3.0 \cdot 10^{-20} \mathrm{~m}^{2} \mathrm{~W}^{-1}$, we find a theoretical value of $\Delta \varphi_{\max } / \Delta L=1.04 \pi / \mathrm{cm}$. This is in surprisingly good agreement with the experimental data.

The effect of the GVD on the temporal profile is a slight stretching or flattening of the pulse and at the same time an almost linear frequency chirp across the central part of the pulse. It is this linear variation of the frequency that enables the use of the anomalous dispersion provided by a grating pair to compress the pulse. Fig. 4 shows the calculated input and output pulses for a 250 fs pulse, together with the calculated frequency chirp.

\subsection{Pulse compression}

The large spectral bandwidth obtainable through self-phase modulation in the photonic crystal fibre makes it a potentially very interesting medium for pulse compression. The non-uniform spectrum obviously implies that a compressed pulse will have a degree of frequency chirp and ringing, so we might anticipate a somewhat larger value for the time-bandwidth product for the compressed pulse than for the initial laser pulse. 
The pulse compression is easily incorporated in the model by introducing negative dispersion after the fibre. If the output electric field from the fibre is denoted $E_{\text {out }}(t)$ its intensity spectrum is $\left|E_{\text {out }}(\omega)\right|^{2}$, where $E_{\text {out }}(\omega)$ is the Fourier transform of $E_{\text {out }}(t)$. The effect of the negative dispersion is a frequency dependent phase shift of the spectrum:

$$
E_{d c}(\omega)=E_{\text {out }}(\omega) \exp \left(-i a_{c}\left(\omega-\omega_{0}\right)^{2}\right)
$$

where $\omega_{0}$ is the centre frequency and $a_{c}$ the introduced group delay dispersion.

The temporal profile, $E_{\mathrm{dc}}(t)$, of the compressed pulse can now be determined as the Fourier transform of $E_{\mathrm{dc}}(\omega)$ and the corresponding intensity distribution is $I_{\mathrm{dc}}(t)=\left|E_{\mathrm{dc}}(t)\right|^{2}$. The distribution and its peak intensity are both functions of the dispersion compensation parameter $a_{c}$. An optimum value for $a_{c}$ is taken to be the value, $a_{c, \text { opt }}$, at which the peak intensity of the pulse is maximised. Fig. 4 shows the calculated profile of the pulse at the relevant optimum value for the particular case under consideration. The relatively low group velocity dispersion leads to a pulse shape similar to the zero-GVD case [15]. The pulse has a narrow central peak with a FWHM of 17 fs containing $75 \%$ of the pulse energy. The remaining energy is mainly in two side lobes immediately adjacent to the central peak. This general pulse shape is found for all values of $\Delta \varphi_{\max } / \Delta L$ above approximately $0.2 \pi / \mathrm{cm}$. Although the width of the central peak varies as shown in Fig. 5 , the power in the peak remains constant at $75 \%$. Fig. 5 also shows the optimum dispersion compensation required as a function of $\Delta \varphi_{\max } / \Delta L$. Both curves are approximately inversely proportional to the transmitted power.

The power transmitted through the fibre was varied in the range 50 to $215 \mathrm{~mW}$. For each power the value of $\Delta \varphi_{\max } / \Delta L$ can be estimated by comparing the recorded spectrum with the calculated spectra. We find that $\Delta \varphi_{\max } / \Delta L$ is approximately $6.8 \pi / \mathrm{cm}$ per Watt of transmitted power. For each power level the optimum grating separation was also determined, 
i.e. the separation at which the autocorrelation signal was maximum. The corresponding anomalous dispersions are shown in Fig. 5. The data points are consistently approximately $0.4 \times 10^{-3} \mathrm{ps}^{2}$ higher than the calculated dispersions. This is due to the model not fully accounting for the dispersion in the optical system. First of all it is based on an estimated value of $20 \mathrm{ps} / \mathrm{nm} \cdot \mathrm{km}$ for the fibre dispersion based on the general shape of the spectra. A value of $30 \mathrm{ps} / \mathrm{nm} \cdot \mathrm{km}$ (corresponding to a group delay dispersion of $11 \mathrm{ps}^{2} / \mathrm{km}$ ) would not alter the spectra severely, but the additional dispersion will shift the theoretical curve up by $\sim 0.35 \times 10^{-3}$ $\mathrm{ps}^{2}$. Secondly, we are operating with an up to $100 \mathrm{~nm}$ wide pulse centred about $70 \mathrm{~nm}$ away from zero-dispersion point, so higher order dispersion will inevitably play a role. Finally, dispersion in the lens used to collimate the fibre output is not accounted for in the model.

\subsection{Autocorrelation measurements}

In order to study the pulse shape in detail we record the interferometric autocorrelation signal for the pulse as described above. Fig. 6 shows an example of the recorded trace for $175 \mathrm{~mW}$ transmitted through the fibre at a wavelength of $830 \mathrm{~nm}$. The grating separation was chosen to maximise the peak intensity or, equivalently, the autocorrelation signal. The signal displays the familiar symmetric shape with a peak to background ratio of $8: 1$. The notable difference compared with the familiar sech ${ }^{2}$ autocorrelation signal is the prominent structure in the wings due to the side lobes on the pulse.

The autocorrelation function for a laser pulse, $E(t)$, can be calculated as:

$$
A(\Delta t)=\int_{-\infty}^{\infty}\left|(E(t)+E(t+\Delta t))^{2}\right|^{2} d t
$$


where $\Delta t$ is the time delay between the two arms of the interferometer. However, there is no unique way of inferring the exact pulse shape from the autocorrelation signal [18]. The ratio of the FWHM of a pulse and the number of interference fringes across the autocorrelation trace is in general a function of the pulse shape. We have used the calculated profiles $E_{\mathrm{dc}}(t)$ to determine the theoretical autocorrelation functions. Fig. 7 shows the calculated signal for the optimum dispersion compensation $a_{\mathrm{c}, \mathrm{opt}}$, and the signals for $a_{\mathrm{c}, \mathrm{opt}} \pm 10 \%$ normalised to the maximum signal for $a_{\mathrm{c}, \mathrm{opt}}$. The three autocorrelation signals have the same general shape. The width is only weakly affected, but the amplitude drops by $>35 \%$ on the $10 \%$ change in dispersion. At the more detailed level we observe that the structure in the wings of the autocorrelation signal are more prominent for $a_{c}>a_{c, \text { opt }}$. These features are also recognised in the experimental data shown in Fig. 8. This figure shows the recorded autocorrelation traces for a range of grating separations around the optimum point.

A significant difference between the theoretical and experimental results, however, is the fact that the calculated signal for the 17 fs pulse has $\sim 9$ fringes at FWHM while the experimental signal in Fig. 6 has $\sim 13.5$. This is most likely due to our inability to implement an exact dispersion compensation. For bandwidth in the tens of $\mathrm{THz}$ range, the third order dispersion in both fibre and grating pair would be expected to play a significant role.

We have calculated the FWHM for an optimally compressed pulse and the corresponding autocorrelation functions for a range of pulse bandwidths. We find that the ratio of the FWHM of a pulse and the number of interference fringes across the autocorrelation trace is a constant $1.84 \mathrm{fs} /$ fringe or slightly larger than the ratio of $1.44 \mathrm{fs} / \mathrm{fringe}$, that we can calculate for $\operatorname{sech}^{2}$ pulse. The 13.5 fringes observed in the experimental data shown in Fig. 6 therefore correspond to a pulse width of $24.8 \mathrm{fs}$ or an order of magnitude compression of the original laser pulse. 
These measurements were carried out with a fibre length of $86 \mathrm{~mm}$. This was chosen to be short enough that the broadened spectrum did not extend into the region of anomalous dispersion. If this happens we observe clear evidence, both spectrally and in the autocorrelation trace, that solitons start forming and the pulse breaks up. However, even before this happens one might expect the higher order dispersion of the combined fibre and grating system to be a significant limiting factor. We have not attempted to incorporate this in the model.

\subsection{The time-bandwidth product}

The time-bandwidth product is a relatively high 0.97 for the $830 \mathrm{~nm}$ pulses compared with 0.32 for a sech ${ }^{2}$ pulse, 0.44 for a Gaussian pulse, and 0.66 using the results of our model. This is in part due to the non-linearity of the frequency chirp across the pulse. Increased dispersion in the fibre would tend to produce a less broadened pulse with a more linear chirp. Indeed, for a pulse at $810 \mathrm{~nm}$ we observe a $29 \mathrm{THz}$ wide spectrum which compresses into a $29 \mathrm{fs}$ pulse. This corresponds to a time-bandwidth product of 0.84 .

A longer section of fibre would also tend to linearise the chirp and result in a lower timebandwidth product. However, in order for this to work well the spectral broadening would also have to be reduced either by reducing the non-linearity of the fibre or by dropping the pulse energy. Two similar fibres with larger core sizes and longer zero-dispersion wavelengths were also used in the same set-up. Despite displaying narrower spectra and relatively longer pulses due to the lower non-linearity and the larger dispersion we observed an overall reduction in the time-bandwidth product with these fibres. For a $215 \mathrm{~mm}$ long fibre with a $3.5 \mu \mathrm{m}$ core and a zero dispersion point at $\sim 975 \mathrm{~nm}$ we observed a spectral width of $18 \mathrm{THz}$ and a pulse width of 
$43 \mathrm{fs}$. This corresponds to a time-bandwidth product of 0.77 . The even lower non-linearity of a $200 \mathrm{~mm}$ section of a $4.7 \mu \mathrm{m}$ core with zero dispersion at $1040 \mathrm{~nm}$ results in a time-bandwidth product of 0.73 .

\section{Optimisation of the PCF pulse compressor}

The optimum condition for pulse compression was determined theoretically by Tomlinson et al. [15]. The two important fibre parameters determining the performance of a fibre compressor are the non-linear length $L_{\mathrm{NL}}$ and the dispersion length $L_{\mathrm{D}}$ :

$$
\begin{gathered}
L_{N L}=\frac{1}{\gamma P_{0}} \\
L_{D}=\frac{T_{0}^{2}}{\left|\beta_{2}\right|}
\end{gathered}
$$

where $\beta_{2}$ is the group velocity dispersion. The optimum compression factor $F_{c}=T_{0} / T_{c}$, where $T_{\mathrm{c}}$ is the duration of the compressed pulse, was found to be [15]:

$$
F_{c}=0.63 \sqrt{\frac{L_{D}}{L_{N L}}}=0.63 T_{0} \sqrt{\frac{\gamma P_{0}}{\left|\beta_{2}\right|}}
$$

This expression immediately shows the advantage of using the photonic crystal fibre for the pulse compressor. The non-linearity represented by the factor $\gamma$ is high and the dispersion is relatively low, resulting in a large compression factor. The corresponding optimal fibre length is [15]: 


$$
L_{\text {opt }}=0.8 \pi \sqrt{L_{D} L_{N L}}=\frac{0.8 \pi T_{0}}{\sqrt{\gamma P_{0}\left|\beta_{2}\right|}}
$$

For the relevant fibre parameters for our experiment $\left(\gamma P_{0}=1.1 \pi / \mathrm{cm}, \beta_{2} \approx 7 \mathrm{ps}^{2} / \mathrm{km}\right.$, and $T_{0}=250 \mathrm{fs}$ ) we find that $L_{\mathrm{D}}$ is approximately $9 \mathrm{~m}$ while $L_{\mathrm{NL}}$ is a mere $2.9 \mathrm{~mm}$. This leads to a maximum compression factor of 35 , which would be obtained at a fibre length of $40 \mathrm{~cm}$. The observed order of magnitude compression achieved with a fibre length of less than a quarter of this is in good qualitative agreement with this theory.

Tomlinson et al. [15] also showed that the quality of the pulse depends on the fibre length and is best at $L_{\text {opt. }}$ In the current experiments we observed a reduction in the timebandwidth product, equivalent to an improvement in the pulse quality, by going to shorter wavelengths. This is due to the larger $\beta_{2}$ resulting in a smaller $L_{\mathrm{opt}}$ and hence the experiment operated closer to the optimum condition. At the same time we expect a slight reduction in $F_{\mathrm{c}}$ and hence a longer compressed pulse.

As mentioned above, we were forced to use a shorter than optimal length of fibre as the bandwidth of the pulse would otherwise extend into the anomalously dispersive regime. Assuming a time-bandwidth product of order unity we find that the bandwidth, $\Delta v_{c}$, at the output of the fibre will have to be of order:

$$
\Delta v_{c} \approx \frac{1}{T_{c}}=\frac{F_{c}}{T_{0}}
$$

With an $F_{\mathrm{c}}$ value of 35 and a 250 fs input pulse, this would correspond to a spectral broadening of the output in excess of $300 \mathrm{~nm}$. 
The high compression factor obtainable with a photonic crystal fibre is partly due to the relatively small group velocity dispersion. This, in turn, is at least partly due to the laser operating near the zero-point for the dispersion. In our experiments with the $2.6 \mu \mathrm{m}$ diameter fibre, this is somewhat less than $\Delta v_{c} / 2$, hence we used a shorter than optimal fibre. However, the dispersive properties of photonic crystal fibres can to some extent be tailored [11] offering the enticing prospect of designing a fibre structure optimised for pulse compression. The fibre would have a small and highly non-linear core and a relatively small, positive, and as near as possible constant group velocity dispersion over a bandwidth of a few hundred nm [19]. With such a design the PCF would be far superior to standard step-index fused silica fibre for the purpose of ultra-short pulse compression.

\section{Conclusion}

We have demonstrated the use of a photonic crystal fibre, in combination with a grating pair, for pulse compression of $250 \mathrm{fs}$ Ti:Sapphire laser pulses to below $25 \mathrm{fs}$. The set-up is fairly basic and no attempt has been made to compensate higher order dispersion in the fibre or the grating pair. Even so, good qualitative agreement was obtained with a numerical model describing the propagation and broadening of the pulse through the fibre.

The current experiment was carried out with a fairly standard PCF design intended for white-light continuum generation. The third order dispersion and the proximity to the zero-point for the GVD are clearly limiting factors in the experiment. However, the PCF offers the possibility of designing non-linear fibres with a relatively flat dispersion over a wide and selectable wavelength range. Indeed our model indicates that pulse compression to below 10 fs would be achievable in such a fibre. The notable difference between this approach and one 
based on standard silica fibre, however, is that this technique is a universally applicable add-on to a standard laser system and does not require further pulse amplification. Thus, this technique would then offer a convenient way of transforming the outputs of widely available commercial femtosecond laser systems to the few cycle regime, where direct operation of lasers is rather more involved.

The main penalty is that $25 \%$ of the power is outside the narrow central part of the pulse. However, this is not significant for applications where it is mainly the peak intensity, that is of importance. One such application is multi-photon microscopy [20]. In this technique, two or more photons are absorbed simultaneously by a prepared sample and the resultant fluorescence is used to obtain information regarding the structural composition. One of the most important features of the excitation source is a sufficiently high peak power, whilst retaining a modest average power. The pulse compression characteristics afforded by the PCFs would therefore significantly enhance the capabilities of existing commercial laser sources for high-resolution multi-photon fluorescence microscopy.

\section{Acknowledgements}

This work was carried out in collaboration with NKT Research and Innovation A/S and Crystal Fibre A/S, from whom the fibre samples were provided. Valuable discussions with Alison Gurney, Allister Ferguson and Nigel Langford are also acknowledged.

\section{References}


1. G.P. Agrawal: Nonlinear Fiber Optics (Academic, San Diego, 1995)

2. L.F. Mollenauer, R.H. Stolen, and J.P. Gordon: Phys. Rev. Lett. 45, 1095-1098 (1980)

3. H. Nakatsuka, D. Grischkowsky, and A.C. Balant: Phys. Rev. Lett. 47, 910-913 (1981)

4. C.V. Shank, R.L. Fork, R. Yen, R.H. Stolen, and W.J. Tomlinson: Appl. Phys. Lett. 40, 761$763(1982)$

5. E.P. Ippen, H.A. Haus, and L.Y. Liu: J. Opt. Soc. Am. B6, 1736-1745 (1989)

6. A.M. Johnson, R.H. Stolen, and W.M. Simpson: Appl. Phys. Lett. 44, 729-731 (1984)

7. R.L. Fork, C.H. Brito Cruz, P.C. Becker, and C.V. Shank: Opt. Lett. 12, 483-485 (1987)

8. S. Lakó, J. Seres, P. Apai, J. Balázs, R.S. Windeler, R. Szipőcs: Appl. Phys. B76, 267-275 (2003)

9. T. Südmeyer, F. Brunner, E. Innerhofer, R. Paschotta, K. Furusava, J.C. baggett, T.M. Munro, D.J. Richardson, and U. Keller: Opt. Lett. 28, 1951-1953 (2003)

10. J.C. Knight, T.A. Birks, P.St.J. Russell and D.M. Atkin: Opt. Lett. 21, 1547-1549 (1996)

11. T.A. Birks, J.C. Knight, and P.St.J. Russell: Opt. Lett. 22, 961-963 (1997)

12. J.K. Ranka, R.S. Windeler, and A.J. Stentz: Opt. Lett. 25, 25-27 (2000)

13. J.K. Ranka, R.S. Windeler, and A.J. Stentz: Opt. Lett. 25, 796-798 (2000)

14. A.V. Husakou and J. Herrmann: Phys. Rev. Lett. 87, 203901-203904 (2001)

15. W.J. Tomlinson, R.H. Stolen, and C.V. Shank: J. Opt. Soc. Am. B1, 139-149 (1984)

16. J.K. Ranka, A.L. Gaeta, A. Baltuska, M.S. Pshenichnikov, and D.A. Wiersma: Opt. Lett. 22, 1344-1346 (1997).

17. K.P. Hansen, J. Riis Jensen, D. Birkedal, J.M. Hvam, and A. Bjarklev: Optical Fiber Communications Conference, 2002 OSA Technical Digest Series (Optical Society of America, Washington, D. C., 2002), paper ThGG8 
18. K. Naganuma, K. Mogi, and H. Yamada: IEEE J. Quant. Elec. 25, 1225-1233 (1989)

19. A. Ferrando, E. Silvestre, J.J. Miret, and P. Andrés: Opt. Lett. 25, 790-792 (2000)

20. W. Denk, J.H. Stickler, and W.W. Webb: Science 248, 73-76 (1990) 


\section{Figure captions:}

Fig. 1. The Experimental set-up. The output of a mode-locked Ti:Sapphire laser is sent through a Faraday Isolator (FI) and coupled into a section of photonic crystal fibre (PCF). The spectrally broadened output is compressed by the negative dispersion of a grating pair. The interferometric autocorrelation trace of the resulting pulse is recorded.

Fig. 2. The spectrum of the light transmitted through $86 \mathrm{~mm}$ of fibre at an average transmitted power of $188 \mathrm{~mW}$. The spectrum shows a symmetric and approximately $38 \mathrm{THz}$ wide distribution (FWHM) with 9 individual maxima. The same width and similar structure can be modelled by pure self-phase modulation (dotted curve) but only for an unrealistically large initial pulse width of $315 \mathrm{fs}$. By including a $20 \mathrm{ps} / \mathrm{nm} \cdot \mathrm{km}$ group velocity dispersion in the model we obtain the same width and similar structure for a pulse length of $270 \mathrm{fs}$ and assuming a phase shift on the peak of the pulse, $\Delta \varphi_{\max } / \Delta L$, of $1.10 \mathrm{~m} / \mathrm{cm}$ (smooth solid curve).

Fig. 3. The FWHM of the spectral distribution of the fibre output varies linearly with the transmitted power. The fibre length is $86 \mathrm{~mm}$.

Fig. 4. Calculated distributions for pulse compressor. The input pulse is assumed to be a $250 \mathrm{fs}$ FWHM sech ${ }^{2}$ pulse. The output profile and frequency deviation is calculated assuming an 86 $\mathrm{mm}$ long fibre with a group velocity dispersion of $20 \mathrm{ps} / \mathrm{nm} \cdot \mathrm{km}$ and a phase shift on the peak of the pulse, $\Delta \varphi_{\max } / \Delta L$, of $1.10 \mathrm{~m} / \mathrm{cm}$. The intensity is normalised to the peak intensity of the input pulse. The almost linear chirp of the frequency across the pulse allows compression of the 
pulse using the negative dispersion of a grating pair. The optimally compressed pulse shown here is $\sim 17$ fs long with $\sim 75 \%$ of the power in the central feature. The dispersion required to obtain this compression is $1.22 \times 10^{-3} \mathrm{ps}^{2}$.

Fig. 5. Calculated optimum dispersion for the grating pair and the corresponding length of the compressed pulse as a function of fibre non-linearity. The dots are the calculated values for the FWHM of the pulse while the solid curve is the calculated dispersion. In the absence of group velocity dispersion in the fibre both curves would have been hyperbolae. The squares are the measured dispersions for transmitted powers in the range $50-215 \mathrm{~mW}$. The offset is due to the model not fully accounting for the dispersion in the system.

Fig. 6. Experimentally recorded autocorrelation signal for an optimally compressed pulse at 175 $\mathrm{mW}$ transmitted power. The $\sim 13.5$ fringes FWHM of this pulse corresponds to a compressed pulse width of $25 \mathrm{fs}$. The dispersion required for this optimum compression is $1.22 \times 10^{-3} \mathrm{ps}^{2}$.

Fig. 7. Calculated autocorrelation traces for the optimum value of the grating dispersion and $\pm 10 \%$ of this value. The curves are normalised to a peak value of 8 for the optimal case.

Fig. 8. Recorded autocorrelation traces for a range of grating separations. The data set taken at the optimum separation of $2.9 \mathrm{~mm}$ is shown in Fig. 6. 


\section{Figures:}

Fig. 1. The Experimental set-up. The output of a mode-locked Ti:Sapphire laser is sent through a Faraday Isolator (FI) and coupled into a section of photonic crystal fibre (PCF). The spectrally broadened output is compressed by the negative dispersion of a grating pair. The interferometric autocorrelation trace of the resulting pulse is recorded.

Fig. 2. The spectrum of the light transmitted through $86 \mathrm{~mm}$ of fibre at an average transmitted power of $188 \mathrm{~mW}$. The spectrum shows a symmetric and approximately 38 $\mathrm{THz}$ wide distribution (FWHM) with 9 individual maxima. The same width and similar structure can be modelled by pure self-phase modulation (dotted curve) but only for an unrealistically large initial pulse width of $315 \mathrm{fs}$. By including a $20 \mathrm{ps} / \mathrm{nm} \cdot \mathrm{km}$ group velocity dispersion in the model we obtain the same width and similar structure for a pulse length of $270 \mathrm{fs}$ and assuming a phase shift

on the peak of the pulse, $\Delta \varphi_{\max } / \Delta L$, of $1.10 \mathrm{~m} / \mathrm{cm}$ (smooth solid curve).
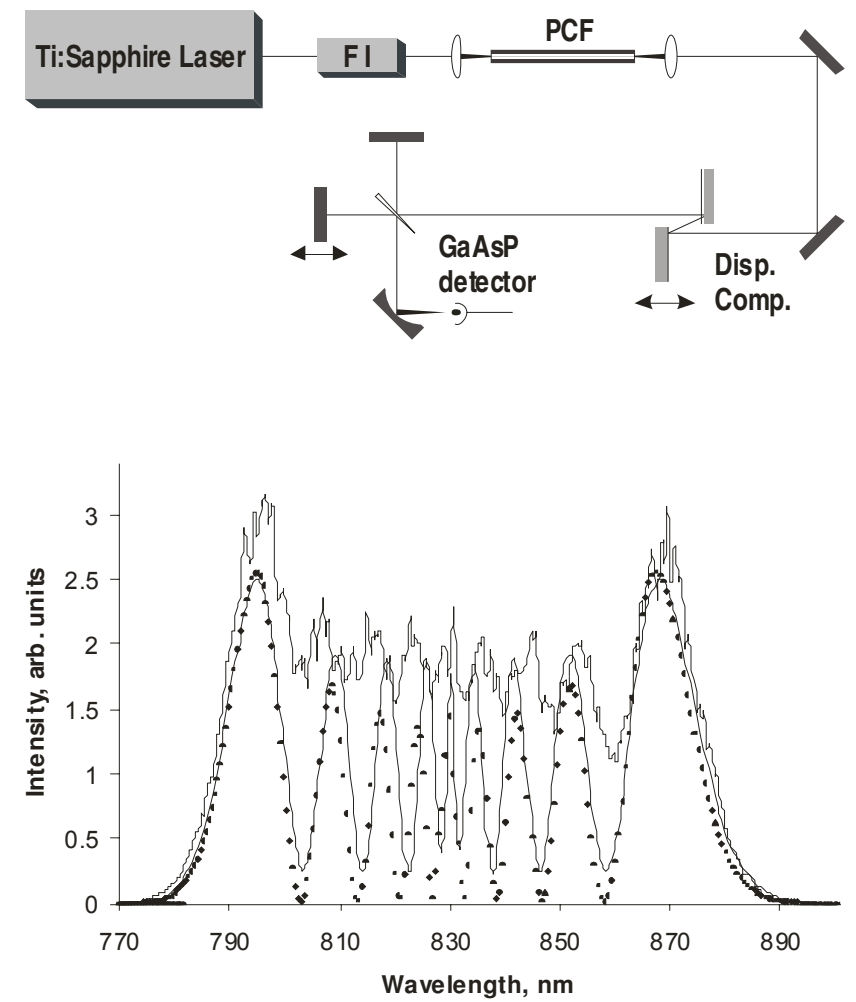

Fig. 3. The FWHM of the spectral distribution of the fibre output varies linearly with the transmitted power. The fibre length is $86 \mathrm{~mm}$.

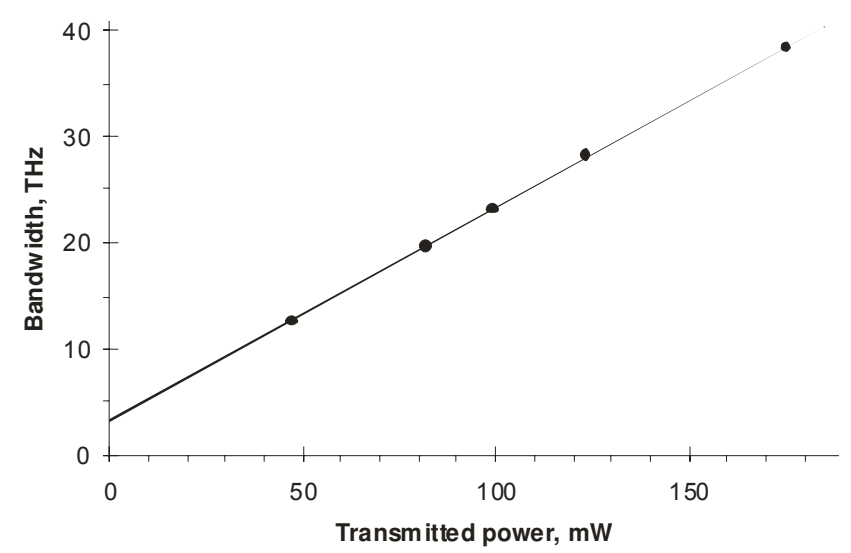


Fig. 4. Calculated distributions for pulse compressor. The input pulse is assumed to be a 250 fs FWHM sech ${ }^{2}$ pulse. The output profile and frequency deviation is calculated assuming an $86 \mathrm{~mm}$ long fibre with a group velocity dispersion of $20 \mathrm{ps} / \mathrm{nm} \cdot \mathrm{km}$ and a phase shift on the peak of the pulse, $\Delta \varphi_{\max }$ $/ \Delta L$, of $1.10 \pi / \mathrm{cm}$. The intensity is normalised to the peak intensity of the input pulse. The almost linear chirp of the frequency across the pulse allows compression of the pulse using the negative dispersion of a grating pair. The optimally compressed pulse shown here is $\sim 17$ fs long with $\sim 75 \%$ of the power in the central feature. The dispersion required to obtain this compression is $1.22 \times 10^{-3} \mathrm{ps}^{2}$.

Fig. 5. Calculated optimum dispersion for the grating pair and the corresponding length of the compressed pulse as a function of fibre non-linearity. The dots are the calculated values for the FWHM of the pulse while the solid curve is the calculated dispersion. In the absence of group velocity dispersion in the fibre both curves would have been hyperbolae. The squares are the measured dispersions for transmitted powers in the range $50-215 \mathrm{~mW}$. The offset is due to the model not fully accounting for the dispersion in the system.

Fig. 6. Experimentally recorded autocorrelation signal for an optimally compressed pulse at $175 \mathrm{~mW}$ transmitted power. The $\sim 13.5$ fringes FWHM of this pulse corresponds to a compressed pulse width of $25 \mathrm{fs}$. The dispersion required for this optimum compression is $1.22 \times 10^{-3} \mathrm{ps}^{2}$.
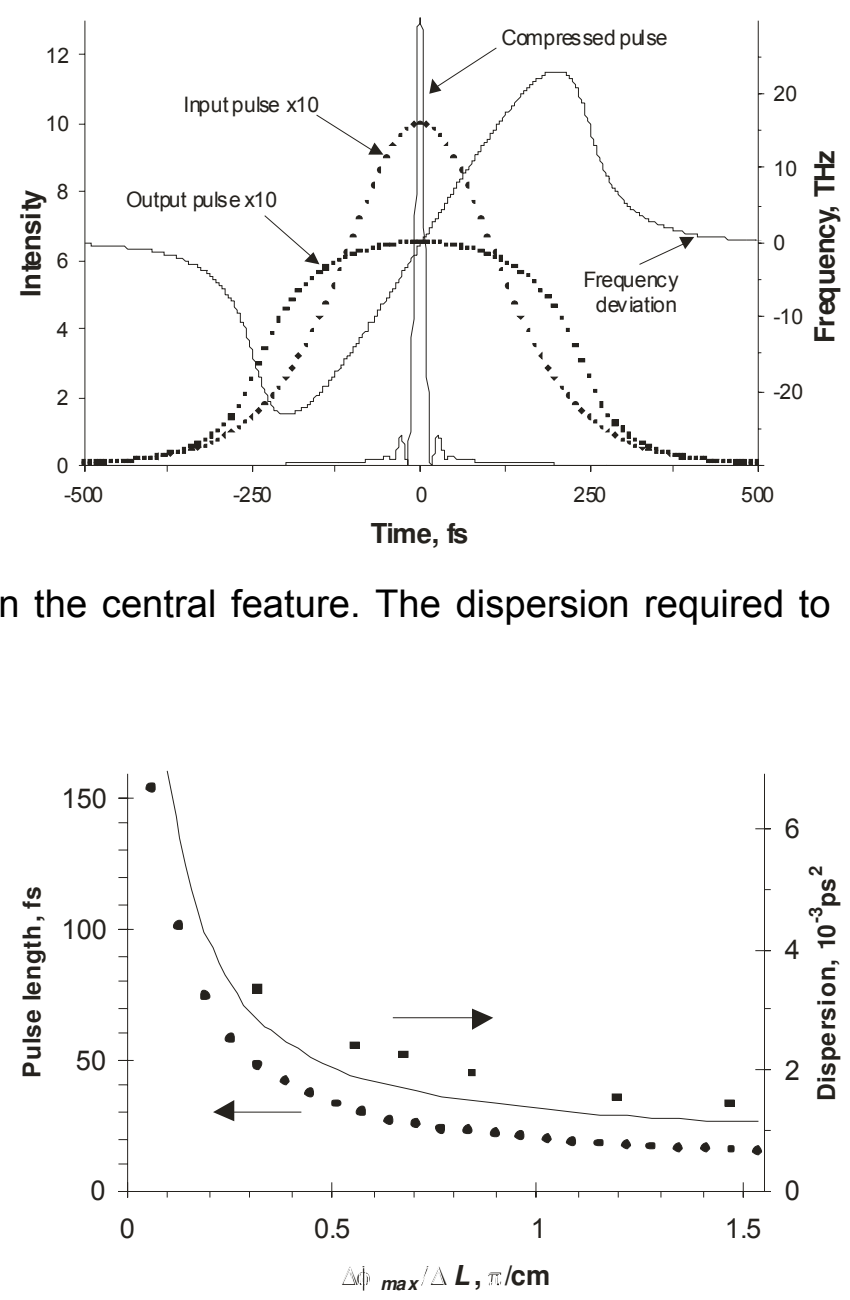

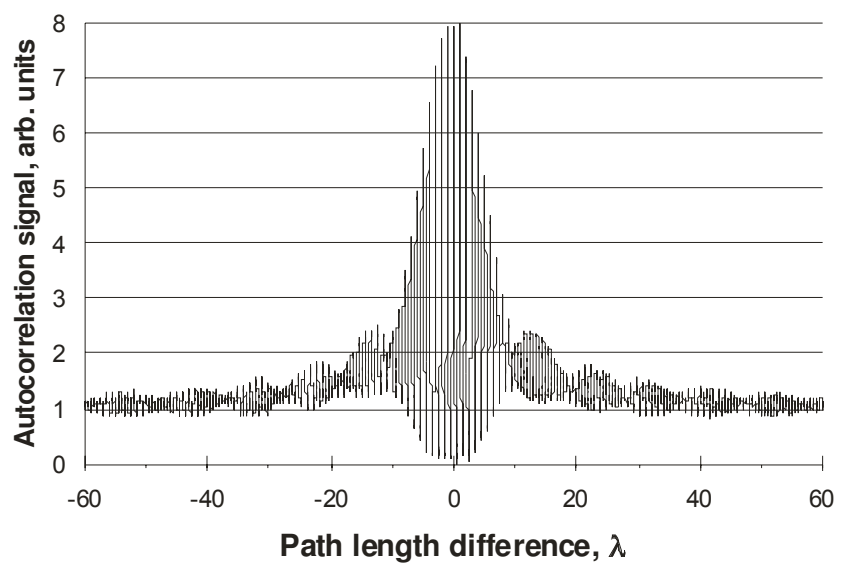


Fig. 7. Calculated autocorrelation traces for the optimum value of the grating dispersion and $\pm 10 \%$ of this value. The curves are normalised to a peak value of 8 for the optimal case.
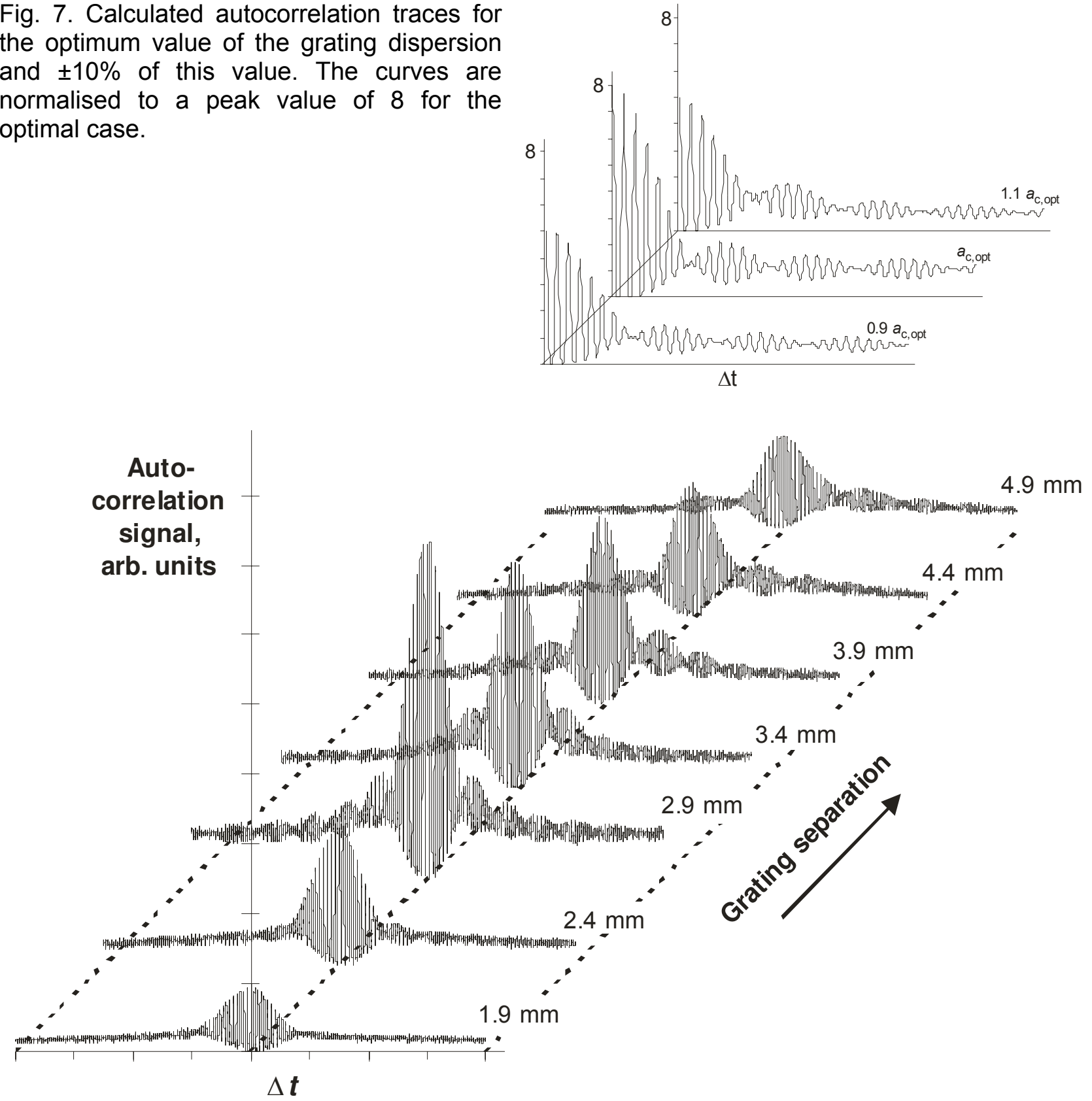

Fig. 8. Recorded autocorrelation traces for a range of grating separations. The data set taken at the optimum separation of $2.9 \mathrm{~mm}$ is shown in Fig. 6 . 